\title{
GOLFE: OS HÁBITOS ALIMENTARES DOS JOGADORES SÉNIORES
}

\author{
AUTORES \\ A. P. Ferreira de Brito ${ }^{1}$ \\ Ruben Gonçalves Pereira ${ }^{2}$ \\ 'Facultade de Ciencias da Educación, \\ Universidade de Vigo, Espanha \\ ${ }^{2}$ Universidade Lusófona do Porto
}

GOLFE: OS HÁBITOS ALIMENTARES DOS JOGADORES SÉNIORES 4(2): 5-10

PALAVRAS-CHAVE

golfe, alimentação, saúde.

\section{INTRODUCÃO}

O objectivo deste estudo é apresentar o contexto que justifica a ligação das performances dos jogadores de golfe com os seus hábitos alimentares. A alimentação influencia inequivocamente a saúde e o desempenho desportivo dos jogadores. Uma ingestão nutricional adequada contribui para optimizar a composição corporal e reservas energéticas em função do desporto. A ausência de estudos nesta área, despoletou o interesse da investigação, de forma a contribuir cientificamente para um aplanar de conhecimentos e uma abertura para a intervenção da abordagem por outras áreas científicas tais como a nutrição.

0 interesse do tema, surgiu da vivência com os jogadores de golfe e da modalidade em si, que embora sendo um tema importante no nosso estudo, poderá ser apenas um indicador da performance.

\section{METODOLOGIA}

Este estudo exploratório configurou-se a partir da caracterização dos hábitos alimentares dos jogadores de golfe com idades iguais ou superiores a 50 anos, bem como em observações e medições directas dos campos e jogadores. Os jogadores foram avaliados momentos antes da competição e as seguintes variáveis determinadas: peso, estatura, envergadura, quatro pregas cutâneas (bicipital, tricipital, subescapular, supraespinal], conforme a metodologia proposta. Foram também mensurados os valores da bio impedância e aplicados cardiofrequenciométros durante a volta. No que concerne à constituição da amostra dos jogadores, esta foi do tipo aleatória por aglomerados e, por ser estrategicamente plausível, representativa e viável, optamos por uma população 160 de jogadores, de idade média de 58 anos, em 45 campos de golfe. Os critérios que estiveram subjacentes à selecção dos jogadores para objecto do estudo foram:

- serem jogadores com idade igual ou superior a 50 anos.

- serem sócios da Federação Portuguesa de Golfe e praticantes regulares de golfe.

- terem Handicap homologado pelo respectivo clube.

\section{Procedimentos Estatísticos}

Variáveis descritivas e tabelas de frequência; Testes de independência do Qui - Quadrado; Ró de Spearman e R de Pearson - Medir o grau de dependência das variáveis; $t$ de Student e Anova - Comparação de médias em cada nível de variável; Análise Factorial - Resumo de informação contida num conjunto mais lato de variáveis.

\section{RESULTADOS}

A alimentação pode influenciar positiva ou negativamente o rendimento de um jogador, devendo ser orientada de modo a melhorar a capacidade desportiva e uma boa saúde a longo prazo. Uma selecção adequada dos alimentos, quanto 
à quantidade, composição e momento de ingestão, influencia a saúde e performance dos jogadores (ADA et al., 2000; Maughan \& Burke, 2000). A alimentação dos jogadores de golfe, deverá ser individualizada e adaptada a cada situação, proporcionando a satisfação das necessidades energéticas e fisiológicas, através de um adequado fornecimento em calorias, hidratos de carbono, gorduras, proteínas, água, minerais e vitaminas e, por outro lado, um correcto enquadramento destes alimentos em: ração de treino, ração de competição e ração de recuperação. 0 gasto energético com o exercício depende da natureza, duração, intensidade e frequência deste, e das características do jogador (peso e composição corporal, altura, sexo e idade) (ADA et al., 2000). Uma correcta nutrição promove melhores adaptações ao estimulo do treino, diminui o risco de lesão ou de doença, uma vez que ao manter a função imunológica, colabora na obtenção e manutenção de um peso e composição corporais adequados, preservando as massas ósseas e musculares, modulando a disponibilidade dos substratos energéticos, contribuindo também para melhoria significativa da recuperação após o exercício (ADA et al., 2000).

Analisando os dados que nos são dados a interpretar, deparamos que não diferem da normalidade, uma vez que $76,4 \%$ dos jogadores consideraram um factor determinante uma correcta alimentação. Assim e, considerando que um jogador deverá fazer 5-6 refeições diárias: pequeno-almoço, meio da manhã, almoço, lanche, jantar e eventualmente ceia, os nossos dados encontram similitudes, pois para 47,2\% o número de refeições está distribuído pelas três princi- pais, sendo a refeição fundamental o pequeno-almoço para $75,9 \%$, situando-se para $43,5 \%$ dos jogadores entre as 7 e as 8 horas, em dias de treinos ou torneios.

A alimentação condiciona o rendimento desportivo, para $84,9 \%$ dos jogadores.

A nível de consumo de lacticínios, podemos considerar dentro dos padrões normais, uma vez que os jogadores que não os consomem de uma forma, os ingere de outra. Assim, apenas $13,9 \%$ dos jogadores não tem hábito de ingerir leite e $25,8 \%$ não ingere iogurtes e apenas 3,8\% não inclui nos seus hábitos alimentares o consumo de queijo. É factor de preocupação para $77,9 \%$ dos jogadores a selecção de meio gordo aquando da sua aquisição.

Os jogadores, preferem as suas refeições confeccionadas de modo à obtenção de uma fácil digestão [cozidos e grelhados], promovendo o bom aproveitamento dos seus nutrientes, com pouca gordura (azeite), e que não provoque flatulência, evitando assim o feijão seco, o grão de bico, as favas e a batata.

Fora das refeições, o consumo de bebidas alcoólicas é reduzido sendo a água a bebida de excelência para $87,3 \%$ dos jogadores, sendo que $91,7 \%$ dos jogadores considera fundamental uma correcta hidratação ao longo do dia, tendo de novo a primazia de $95,5 \%$, para saciar a sede. A água é a bebida preferida por $48,4 \%$ dos jogadores, sendo o vinho o eleito de $34,6 \%$ dos jogadores.

As bebidas alcoólicas, são consumidas com moderação $\pm 250 \mathrm{ml}$ de vinho/2 cervejas. As bebidas brancas, são consumidas por vezes com o café, por $46,2 \%$ dos jogadores, porém 34,6\% nunca as ingerem. Dois cafés são a quantidade ingerida por 30,2\% dos jogadores e três cafés por $27 \%$. A ingestão de uma bebida com cafeína em quantidade equivalente às perdas hídricas permite repor apenas $54 \%$ destas e aumentar a excreção de sódio e potássio (Gonzalez-Alonso et al., 1992].

A sopa, é o alimento essencial e preferido por $77,4 \%$ dos jogadores e, 47,2\% afirmam ingerir na maioria das refeições, carne e peixe, na sua dieta semanal.

A carne é a preferida por $30,8 \%$ dos jogadores, sendo porém para $41,5 \%$ dos jogadores incluída tantas vezes carne como peixe nas refei-ções. Na confecção $48,4 \%$ dos joga-dores prefere os alimentos grelha-dos e $44 \%$ cozidos.

0 azeite é a gordura escolhida para $90,5 \%$ dos jogadores, para a confecção dos alimentos:

- Nos grelhados de novo o azeite é o escolhido por $50,6 \%$ dos jogadores optando $21,8 \%$ pelo sumo de limão e manteiga.

- Nas saladas, o tempero tradicional (azeite e vinagre) é utilizado por $63,5 \%$ dos jogadores e somente $34 \%$ utiliza azeite e limão, de referir que $45,6 \%$ dos jogadores ingerem a todas as refeições sala-das ou legumes, e $45 \%$ na maioria das refeições.

A moderação do consumo do sal começa a ser notório, assim 82,5\% dos jogadores não coloca sal após a confecção dos alimentos.

A ingestão de fibras é fundamental para $82,4 \%$ dos jogadores.

As leguminosas são consumidas frequentemente por $48,3 \%$ dos jogadores e $48,8 \%$ inclui por vezes na sua dieta mediterrânica. $\mathbf{O}$ arroz é ingerido na maioria das refeições por $41 \%$ dos jogadores.

Apenas 1,2\% dos jogadores ingerem massas alimentícias a todas as refeições, e os alimentos integrais, estão incluídos nos hábitos alimentares de $61 \%$ dos jogadores. 
0 pão é excluído dos hábitos alimentares de 21,5\% dos jogadores, sendo banido o pão com manteiga por $74,4 \%$ dos jogadores às refeičões.

Relativamente há existência de alimentos que procuram não ingerir antes da competição $88,3 \%$ dos jogadores, não apresenta qualquer impedimento alimentar no período pré competitivo. Antes da competição, um pequeno bife com pouco arroz e salada é a escolha de 58,5\% dos jogadores, massa com bife $30,4 \%$, e, um bom bife com pouco arroz e salada para $11,1 \%$ dos jogadores.

Para 50,9\% dos jogadores dá preferência à fruta relativamente ao doce, e 34,9\% escolhe sempre fruta como sobremesa. Apenas 2,9\% dos jogadores ingerem guloseimas diariamente fora das refeições.

Entre a última refeição e a competição $94 \%$ dos jogadores não ingere alimentos.

Durante a competição, nos tempos mortos, os abastecimentos são realizados por 78,9\% dos jogadores. A toma do abastecimento, acontece sempre para $67,5 \%$ dos jogadores a qual, é executada a meio da competição por 85,7\% dos jogadores, por $4,5 \%$ no final e apenas $1,3 \%$ não realiza qualquer toma. É inquestionável o beneficio da ingestão nutricional durante o exercício físico na melhoria da performance e/ou redução do stress dos sistemas cardiovascular, nervoso e muscular (Casa et al., 2000). Não há uma composição e taxa de ingestão ideais, uma vez que dependerá do stress fisiológico imposto, sendo este em função do exercício, das características do jogador, condições climatéricas e do campo.

A última refeição antes da competição deverá ser uma refeição hipo calórico.
A refeição deverá ser ingerida no mínimo 3 horas e o máximo 4 horas antes da competição.

Se o clima está quente, $86,6 \%$ dos jogadores procura beber mais líquidos. $A$ ingestão voluntária de fluídos repõe apenas 30 a $70 \%$ das perdas hídricas (Hubbard et al., 1984), devendo assim os jogadores basear a sua hidratação em regras e não no seu impulso fisiológico de beber. Um jogador deve iniciar o exercício devidamente hidratados, particularmente quando é inevitável uma desidratação significativa, pelo que se recomenda a ingestão de 400 $-600 \mathrm{~mL}$ de fluídos antes do seu início (Shirreffs et al., 2004).

Durante a competição os jogadores apresentam a necessidade de ingerir bebidas açucaradas, isotónicas e energéticas, com o objectivo de evitar hipoglicémias e proceder à reparação das perdas do organismo provocadas pelo esforço. A presença de concentrações adequadas de sódio numa bebida melhora o seu sabor e estimula a sede, sendo este facto benéfico para a promoção da ingestão voluntária (Wemple et al., 1997). A concentração de sódio habitualmente perdida pela transpiração (20$-80 \mathrm{mmol} / \mathrm{L}$ ] é superior à habitualmente encontrada nas bebidas desportivas (10-25mmol/L), devendo os jogadores ingerir um volume de fluido equivalente a $150 \%$ do peso perdido (Shirreffs et al., 2004). Uma boa hidratação não só é fundamental para o rendimento do jogador, mas também para prevenção de lesões desportivas. A quantidade de água a ingerir depende do trabalho muscular (tipo de campo, percurso a pé/buggy], temperatura, humidade e altitude, sendo considerado ideal $1,5 \mathrm{~L}$ como bebida e 1,5L incorporada nos alimentos (sopa). Durante o exercício, os jogadores são encorajados a ingerir a máxima quantidade de fluidos que tolerem sem desconforto gástrico ate igualarem as perdas hídricas, tendo o cuidado de não as ultrapassarem para não aumentar o peso corporal (Coyle, 2004). 0 consumo de alimentos ou refeições ligeiramente salgadas com água, pode ser ainda mais eficiente para uma correcta rehidratação que a ingestão de bebidas desportivas, água ou ambas (Ray et al., 1998).

Após a competição 43\% ingere bebidas alcoólicas na refeição e $37 \%$, exclui-as da mesma.

Embora uma alimentação equilibrada deva fornecer as quantidades necessárias dos nutrientes básicos, os suplementos podem por vezes ser aconselhados, constatando-se que $82,2 \%$ de jogadores não toma medicamentos vitamínicos, apesar da suplementação pós-exercício de glícidos $(1 \mathrm{~g} / \mathrm{kg})$, diminuir a proteólise, tornando o balanço proteico mais positivo às 24h (Roy et al., 1997). Por atrasar a sensação de fadiga central e servirem como substracto energético, a suplementação com aminoácidos, apresentou resultados inconsistentes em humanos (Blomstrand et al., 1991). A toma produtos farmacêutica para melhorar o rendimento físico é realizada por 4,8\% dos jogadores.

A média do gasto energético numa volta de golfe situa-se entre 622 e $960 \mathrm{Kcal}$ para os 18 buracos, dependendo do traçado do percurso (Magnusson, 1999), estando o valor do nosso estudo, dentro destes valores, uma vez que apresenta uma média de 821 Kcal. Os jogadores no nosso estudo indicam um valor de 104.5 b.p.m, o que nos permite concluir, que o traçado dos campos de golfe portugueses, reúnem as características recomendáveis para a prática da modalidade. 


\section{CONCLUSÃO}

A qualidade dos alimentos, é determinada pelos seus ingredientes químicos, uma vez que são necessários compostos e elementos específicos, para uma nutrição das células individuais. São pelo menos 45 compostos químicos e elementos encontrados nos alimentos sendo considerados essenciais para as células humanas, apesar de os mesmos não serem sintetizados pelo organismo, devendo como tal estarem presentes na dieta evitando assim a doença ou a morte provocada pela sua ausência.

Os glícidos são um nutriente primordial para os jogadores, devendo estar presente em quantidades significativas na sua dieta alimentar [5-12g/ kg/d].

A nível proteico, os jogadores não necessitam de aumentar o seu consumo nos dias de competição, uma vez que os seus hábitos alimentares já superam as recomendações (1,2 a 1,4/7 g/kg/d).

Relativamente aos lípidos, devem apresentar uma contribuição de cerca 20 a $25 \%$ da energia ingerida. Através de uma alimentação equilibrada, os jogadores ingerem as quantidades necessárias para assegurar as exigências energéticas de vitaminas e minerais.

A suplementação não é considerada como factor preponderante não tendo justificação como factor ergogénico.

A alimentação no dia da competição deve apresentar especificidades nutricionais, nomeadamente a nível de glícidos e fluidos visando beneficiar o desempenho e a recuperação dos jogadores.

A ingestão nutricional durante o exercício físico promove a melhoria da performance uma vez que reduz o stress dos sistemas cardiovascular, muscular e nervoso.
A alimentação dos jogadores, deve assim ter características nutricionais especificas, de acordo com o quadro competitivo, torneio, fase de competição, clima, momento de ingestão, entre outros, uma vez que a prioridade do jogador deverá ser a optimização da sua compo-sição corporal e reservas de substractos energéticos e não de um garantir de balanço energético.

A superfície corporal e a actividade física de um indivíduo, determinam as suas necessidades calóricas reais, considerando que a massa magra corporal, a taxa metabólica em repouso e a actividade física, diminuem com o aumento da idade pelo que os jogadores seniores deverão reduzir a ingestão calórica, para compensar estas alteracõos, tendo em atenção que as necessidades dos nutrientes essenciais não diminuem com a idade.

As necessidades calóricas, devem suprir a quantidade de energia necessária para a manutenção das funções fisiológicas do organismo, metabolismo em repouso e as consumidas através da actividade física. (Baker, 1983). Assim as doses diárias recomendadas de ingestão calórica, sugerem uma redução de $10 \%$ em indivíduos a partir dos 50 anos. (Schlienger et al, 1995).

\section{CORRESPONDÊNCIA}

E-mail:

anapaulabrito08@gmail.com; anagolf@netcabo.pt

\section{REFERÊNCIAS}

1. ADA, D.C \& ACSM (2000. Position of the American Dietetic Association, Dietitians of Canada, and the
American College of Sportts Medicine: Nutricion and athletic performance. J Am Diet Assoc, 100(12): : 1543-1556. Allan M. Levy, MD, Mark L. Fuerst. (1999): Golf Injury Handbook: Professional Advice for Amateur Athletes. New York: John Wiley \& Sons, Inc.

2. American College of Sports Medicine. (1990): The Recommended Quantity and quality of Exercise for Developing and Maintaining Cardiorespiratory and Muscular Fitness in Healthy Adults. Med. Sci. Sports Exerc. 22: 265-274.

3. American College of Sports Medicine. (1998): Exercise and physical activity for older adults. Med Sci Sports Exerc. 30: 992-1008.

4. American College of Sports Medicine. (2001): Position Stand on Exercice and Physical Activity for Older Adults. Medicine and Science in Sports and Exercice; 30: $992-$ -1008 .

5. American College of Sports Medicine. (2002): Progression models in resistance training for healthy adults. Med Sci Sports Exerc. 34: : 364-80.

6. Astrand P, O \& Rodahl K (1986): Textbook of Work Psysiology, Physiological Bases of Exercise. New York: McGraw - Hill.

7. Atkins CJ, K Senn, J Rupp, RM Kaplan, et al. (1990): Attendence at health promotion programs: baseline predictors and program outcomes. Health Ed. Q. 17: 417-428.

8. Baker H (1983): Hypovitaminosis in the elderly. Geriatric Medicine Today: 2: 61-6.

9. Baumgartner RN, Heymsfield SB, Lichtman S, Wang J, \& Pierson RN (1991): Body composition in elderly people: Effect of criterion estimates on predictive equations. American Journal of Clinical Nutrition. N.53, pp. 1-9. 
10. Blomstrand E, Hassmen P, Ekblom B \& Newsholme EA (1991). Administracion of branched-chain amino acids during sustained exercise - Effects on performance and on plasma concentration of some amino acids.

11. Bouchard C, Malina R, Pérusse L (1997): Genetics of fitness and physical performance. Champaign, Illinois: Human Kinetics.

12. Brito AP (2007): Categorización e analise do xogo de golfe e dos seus campos en Portugal. Pontevedra: Universidade de Vigo.

13. Carter JEL \& Heath B (1990): Somatotyping - development and applications. Cambridge: Cambridge University Press.

14. Carvalho Helena (2004): Análise Multivariada de Dados Qualitativos - Utilização da HOMALS com o SPSS. 1 ํ Edição. Edições Sílabo, Lda.

15. Casa DJ, Armstrong LE, Hillman SK, Montain SJ, Reiff RV, Rich BSE, Roberts WO, Stone JA (2000): National Athletic Trainers' Association Position Statements: Fluid Replacement for Athletes. Journal of Athletic Training. 35(2): : 212-224.

16. Cervato AM, Pereira FAI, Marucci MFN, Nascimento MI, Gomes MMBC (1997): Apostila: Alimentação na terceira Idade. Faculdade de Saúde Pública da Universidade de São Paulo.

17. Craig FIM \& Salthouse TA (2000): Hanbook of aging and cognition. [ $2^{\mathrm{a}}$ ed.]. Hillsdale, $\mathrm{NJ}$ : Lawrence Erlbaum.

18. Corbin CB, Lindsay R (1994): Concepts of Physical Fitness. lowa: Wcb Brown \& Benchmark Publishers.

19. Costa RF (2001): Composição Corporal: teoria e prática da avaliação. São Paulo: Editora Manole. 20. Coyle EF (2004): Fluid and fuel intake during exercise. Journal of Sports Sciences. 22(1): 39-55.
21. Dobrosielski DA (2002): The metabolic demand of golf in patients with heart disease and in healthy adults: In: Journal Cardiopulmonary Rehabilitation. V. 22(2): : 96-104.

22. Fonseca, Jaime (2001): Estatística Matemática. Vol 2. Lisboa; Edição Sílabo, Lda.

23. Gageiro, João Nunes; Pestana, Maria Helena (2005): Análise de Dados para Ciências Sociais - a complementaridade do SPSS. $4^{\text {a }}$ Edição. Lisboa; Edição Sílabo, Lda. 24. Guedes S, DP \& Guedes JERP (1998): Controle do Peso Corporal. Londrina: Midiograf.

25. Hagberg JM (1990): Exercice, fitness, and hypertension. In Exercice, fitness, and health: A consensus of current knowledge. Champaign: Human Kinetics, pp. 455-66.

26. Hagberg JM, Blair SN, Kaplan N, Ehsani A, Gordon N, Tipton C \& Z, Zambraski E (1993): Position stand: physical activity fitness, and hypertension. Medicine Science Sports Exerc. 25. i-x.

27. Hartz SC (1992): Nutrition in the elderly: The Boston Nutritional Status Survey, SC Hartz, RM Russel and IH Rosenberg [Eds.]. London: Smith-Gordon. P.1-287.

28. Heath B, Carter L (1967): A modified somatotype method. American Journal Phys. Anthropol. 27: : 57-74.

29. Heyward VH \& Stolarczyk LM (2000): Avaliação da composição corporal aplicada. São Paulo: Manole. 30. Heyward HV \& Wagner DR (2004): Applied Body Composition Assessment. Champaign: Human Kinetics.

31. Hubbard RW, Sandick BL, Matthew WT (1984): Voluntary dehydration and alliesthesia for water. Journal of Applied Physiology Respiratory Environmental and Exercise Physiology: 57(3):868-873.
32. Jackson AS, Pollock ML (1978): Generalized equations for predicting body density of men. British Journal of Nutrition; 40: 497-504. 33. Jackson AS, Pollock ML \& Ward A (1980): Generalized equations for predicting body density of women. Medicine and Science in Sports and Exercise: 12: 175-82.

34. Kalache A (1996): Aging and Health. Jour. Aging Physic. Act. 4: : 103-104.

35. Katzel L, Bleecker E, Colman E, Rogus E, Sorkin J \& Golberg A (1995): Effects of weight loss vs. Aerobic exercice training on risk factory for coronary disease in healthy obese, middle-aged and older men. JAMA 274: 1915-20.

36. Katch FI \& Mcardle WD (1996): Nutrição, Exercício e Saúde. (4a ed.) Rio de Janeiro: Medsi.

37. Kim BJ \& Robinson CJ (2005): Postural control and detection of slip/fall initiation in the elderly population. Ergonomics, 48, 1065$-1085$.

38. Kolb M (2000): Motivated Ageing. The Perspectives of Sports Educational Gerontology In: S. Bailey (ed), Perspectives. The Multidisciplinary Series of Physical Education and Sport. Physical Activity and Ageing. pp. 127-134. Meyer e Meyer Sport. 39. Konopka JD (1988): "La alimentación del deportista”. Barcelona; martínez Roca.

40. Kushner RF (1993): Body weight and mortality. Nutrition Reviews. 51: 127-36.

41. Leiker MA, Kandt GK (2001): Physical Activity Associated With Golf Participation: Walking vs Carting. Med. Sci. Sports exerc. 33 [suppl.): $\mathbf{S 5 6 .}$

42. Lindsay DM, Horton JF, Vandervoort A (2000): A Review of Injury Characteristic, Aging Factors and Prevention Programmes for the Older Golfer. Sports Med. 30 (2): : 89-103. 
43. Levy, Allan M. MD \& Fuerst Mark L (1999): Golf Injury Handbook Professional Advice for Amateur Athletes. Jonh Wiley \& Sons, Inc.

44. Lohman TG (1981): Skinfolds body density and their relation to body fatness: a review. Human Biology. 53(2): 181-225.

45. Mcardle WD, Katch FI, Katch VL (1995): Fisiologia do exercício. Energia, nutrição e desempenho humano. Rio de Janeiro. Editora Guanabara.

46. Macheath JA (1984): Activity, Health and Fitness in Old Age. London: Croom Helm.

47. Mahler DA, Froelicher VF, Houston N, York TD (1995): Manual ACSM para la valoración y prescripción del ejercicio. American College of Sports Medicine. Barcelona: Editorial Paidotribo.

48. Martin AD \& Drinkwater DT (1999): Variability in the measures of body: assumptions or techniques?-Sports Medicine.11:277-88. 49. Matsudo SM, Matsudo VR, Barros Neto TL (2000): Impacto do envelhecimento nas variáveis antropométricas, neuromotoras $e$ metabólicas da aptidão física. Revista Brasileira de Ciência e Movimento; 8(4): 21-32.

50. Mazzeo, Robert S; Cavanagn, Peter; Evans, William; Fiatarone, Maria; Hagberg, James; Mcauley, Edward \& Startzel, Jill. (1998): ACSM Position Stand: Exercise and Physical Activity for Older Adults. Med. Sci. Sports. Exerc. V.30, n.6, pp. 992-1008.

51. Magnusson G (1999): Golf: exercise for fitness and health. In: Farrally MR, Cochran AJ, editors. Cience and golf III: proceedings of the World Scientific Congress of Golf. Champaign (IL): Human Kinetics. 51-7.

52. Meijer EP, Westerterp KR, Verstappen FJ (1999): Effect of exercise training on total daily phy- sical activity in elderly humans. Eur. J. Appl. Physiol. 80: 16-21.

53. Mortimer JA, Pirozzolo FJ, Maletta GJ (eds.). (1982): The Aging Motor Sistem. New York: Praeger. 54. Murase YS, Kamie S, Hoshikawa T (1989): Heart Rate and metabolic response to participation in golf: J. Sports Med. Phys. Fitness: 29: : 269-72.

55. Norman V (1995): Exercice Programming for Older Adults. Champaign, Illinois. Human Kinetics.

56. Norton K \& Olds T (1996): Anthropometrica. Australia: University of New South Wales Press. 57. Pate R, Pratt M, Blair N et al. (1995): Physical Activity and Public Health: A recommendation from the Centres for Disease Control and Prevention and the American College of Sports Medicine. Jama: 273: 402-407.

58. Paterson D (1992): Effects of ageing on the cardiorespiratory system. Can. J. Sports Sci., 17(3), 171-177.

59. Pescatello LS and JL VanHeest, Physical activity mediates a healthier body weight in the presence of obesity. Br J Sports Med, 2000. 34(2): pp. 86-93.

60. Pollock ML, Lowenthal DT, Graves JE, Carrol JF (1992): The Elderly and Endurance Training In: JR Sherpard e C Bouchard [eds], Endurance in Sports. 37: 390-406. 61. Pollock ML \& Wilmore JH (1993): Exercícios na Saúde e na Doença. (2 ${ }^{\text {a }}$ ed.). Rio de Janeiro: Medsi.

62. Pollock ML (1995): Exercise Prescription for the Elderly In: WW Spirduso e HM Eckert [eds.). Psysical Activity and Aging. Champaign, Illinois: Humain Kinetics.

63. Rogers MA, Hagberg JM, Martins WH, Ehsani AA, Holloszy JO (1990): Decline in VO2 max With Aging in Masters Athletes and Sedentary Men. J. Appl. Phys. 68: : 2195-2199.
64.Rowland TW(1996): Developmental Exercise Physiology. 8: 117-135. 65. Rosenberg IH, (1997): Nutrition and senescence. Nutr. Rev.: 55 (1pt2): S69-S77.

66. Ross R, Jackson A (1990): Exercise Concepts and Calculations. Benchmark Press Inc.

67. Salthouse TA (1991): Theoretical perspectives on cognitive aging. New Jersey; Lawrence Erlbaum.

68. Sarsfield Cabral, José A., Guimarães, Rui Campos (1997): Estatística. Edição Revista; Mcgraw Hill de Portugal, Lda.

69. Schlienger JL, Pradignac A, Grunenberger F (1995): Nutrition in the elderly: a challenge between facts and needs. Horm Res.43(1-3):46-51. 70. Shepard RJ (1997): Aging, physical activity and health. Champaign, III: Human Kinetics.

71. Siri WE (1961): Body composition from fluid spaces and density: analysis of methods. In: Brozek J \& Henschel A - Techniques for measures body composition. Washington: National Academy of Sciences.

72. Spirduso WW (1995): Physical Dimensions of Aging. Champaign, Illinois: Human Kinetics.

73. Stover C, Stolz J (1996): Golf for the Sénior Player. Clin. Sports Med. 15: 163-178.

74. Thériault G, Lacoste E, Gaboury M, Etal. (1996): Golf injury characteristisc: asurvey from 528 golfers (abstrat); Med. Sci. Sports Exerc.; 28: 5389.

75. Thériault G, lachance P(1998): Golf Injurie: na Overviw. Sports Med. 26(1): 43-57.

76. Wemple RD, Morocco TS, Mack GW (1997): Influence of sodium replacement on fluid ingestion following exercise-induce dehydration. International Journal of Sport Nutrition and Exercise Metabolism: 7(2): 104-116. 